Digital Press Social Sciences and Humanities

Domination inconscience (Id) du personnage principal dans le roman de Thérèse Raquin par Émile Zola

Theresia Damai Ratri and Suma Riella Rusdiarti

Proceeding of Conférence internationale sur le français 2018

Joesana Tjahjani, Merry Andriani, Sajarwa, Wening Udasmoro (eds) 


\title{
Domination inconscience (Id) du personnage principal dans le roman de Thérèse Raquin par Émile Zola
}

\author{
Theresia Damai Ratri* et Suma Riella Rusdiarti \\ Universitas Indonesia, Depok, Indonesia \\ *e-mail : theresia.damai@ui.ac.id
}

\section{Résumé}

Cette communication traite de la domination du Ça dans la vie de Thérèse Raquin qui est le personnage principal du roman Thérèse Raquin d'Émile Zola. La méthode utilisée dans cette communication est une méthode qualitative d'étude de la littérature structurale à l'aide du concept de personnalité structurelle selon Sigmund Freud. L'analyse montre qu'il y a un développement de la personnalité qui conduit à la domination du Ça dans la vie de Thérèse Raquin. La domination du Ça dans la vie de Thérèse a était commencé depuis qu'elle a rencontré Laurent, motivée par le déséquilibre du Moi depuis son plus jeune âge et l'influence de Laurent. La domination du Ça dans l'inconscient révèle les côtés libidinaux, sauvages et primitifs de Thérèse, séparés de tout lien moral et social.

\section{Mots-clés}

Caractère, domination, Freud, le Ça, inconscient

\begin{abstract}
This article talks about unconscious domination (Id) in the life of principal character in the novel Thérèse Raquin by Émile Zola, naturalist French writer. The method used in this research is qualitative method using structural theory and structural personality by Sigmund Freud. The result shows that there's character development that utilize unconscious domination in Thérèse Raquin's life. Thérèse's unconscious domination had started since he met Laurent, motivated by her unequal ego since her childhood and Laurent' influence that's also controlled by Id since his school time.
\end{abstract}

\section{Keywords}

Characters, domination, Freud, unconscious.

\section{Introduction}

En Europe, depuis le 18ème siècle, les romans sont devenus l'œuvre la plus dominante des autres œuvres littéraires. Soutenu par un renforcement de l'éducation de la classe moyenne et la création d'une imprimerie, le roman est devenu une réflexion sociale et historique, le miroir d'une nouvelle civilisation plus moderne (Klarer, 2004, p. 11). Dans le cadre d'une Europe en développement, la France a également connu d'une révolution industrielle au XIXe siècle, à savoir la transformation d'une société agricole en une société industrielle, ainsi que la migration rurale vers les centres industriels. Ce changement de situation sociale a eu l'impact majeur sur la littérature française, dont l'émergence de romans avec des récits qui ont évoqué la vie de la classe moyenne inférieure, qui évoquait auparavant le thème de la bourgeoisie française. De grands auteurs apparaissent également, dont Émile Zola qui fait la promotion de la littérature.

En tant qu'auteur du naturalisme, Émile Zola décrit également le quotidien des gens de cette époque dans ses œuvres. Par exemple, dans Germinal, une histoire des ouvriers du siècle après la révolution industrielle et Thérèse Raquin, histoire d'une vie de famille simple au 19ème siècle. Zola a recueilli des histoires à partir d'observations et d'expériences, puis les a transmises selon la réalité existante 
(Shivanandan, 2007, p. 514). Le flux du naturalisme se concentre sur la représentation vivante des effets sociaux et environnementaux qui affectent le caractère (Klarer, 2004, p. 71). Un exemple que nous pouvons voir se trouve dans la préface du roman Thérèse Raquin. Zola a écrit qu'il a créé la pièce du protagoniste et l'a présentée dans chaque chapitre, des études d'étranges cas psychiatriques (Zola, 1968, pp. 6-7).

Thérèse Raquin raconte l'histoire d'une femme s'appelle Thérèse, élevée par sa tante Mme Raquin depuis l'âge de deux ans. Sa mère n'a jamais été connue, mais prétendument une fille d'Oran; alors que son père était un soldat qui mort en Afrique quelques années après avoir quitté Thérèse. Le fils unique de madame Raquin, Camille, était une personne facilement malade et très gâtée par sa mère. Thérèse devait prendre les mêmes médicaments que Camille et rester à la maison comme une personne malade. Elle a été éduquée pour être une enfant calme et obéissante qui ne savait pas comment exprimer ses pensées et ses désirs. À 21 ans, elle s'est mariée à Camille. Grâce à l'ambition de Camille, la famille a déménagé à Paris. Là, la vie de Thérèse a changé, elle a appris petit à petit à pouvoir refuser de faire ce qu'elle ne voulait pas. Surtout, quand elle a rencontré Laurent, l'ami d'enfance de Camille qui était fort et viril, Thérèse et Laurent ont été des amants et ils ont tué Camille. Un an après la mort de Camille, ils se sont mariés, mais ont été hantés par la crainte que l'un d'eux trahisse. Aussi, ils ont été poursuivis par des cauchemars sous la forme du fantôme de Camille. Devant Mme Raquin qui était en paralysie totale, ils ont révélé leurs actes cruels, mais cela n'a pas réduit leur peur. À la fin, ils ont décidé que la mort était la meilleure solution. Les deux couples sont morts par poison devant madame Raquin qui attendait ce moment.

Ma communication se concentrera sur la dominance de l'aspect inconscient / Le Ça pour les actions des personnages de Thérèse et leurs causes. La méthode de recherche qui sera utilisée dans cette recherche est une méthode qualitative avec une approche littéraire structurelle renforcée par le concept de trois aspects de la personnalité de Freud, à savoir Le Ça, Le Moi et Le Surmoi. Selon Freud dans son livre The Ego and The Id en 1923, il y a trois conditions de l'esprit humain, qui sont conscientes (conscientes / Cs), préconscientes (préconscient / Pcs) et inconscient (inconscient / Ucs). En outre, il existe trois types de psychisme humain, à savoir Le Ça, Le Moi et Le Surmoi.

Le Ça est étroitement lié à l'instinct humain, qui tend à satisfaire ses désirs et évite l'inconfort. Le Ça est la forme primitive d'une personne ainsi que la première à apparaître chez l'homme. Selon Freud, Le Ça est dans le subconscient et n'a aucun contact avec la réalité (le monde conscient). Contrairement à Le Ça, il y a le Surmoi. C'est le Moi idéal qui décrit le comportement humain le plus élevé et qui connaît le bien et le mal. Le Surmoi est très lié à la connaissance, à l'éducation et à la moralité de la personnalité. De plus, il existe le Moi étroitement lié à la perspective d'un individu. Le Moi est un forme d'efficacité et d'activité humaine. C'est une alternative au Ça et le Surmoi. Le Moi chargé de trouver une solution remplis les souhaits du Ça, mais ne viole pas les restrictions imposées par le Surmoi. Le Moi est dans l'état naturel de l'esprit humain conscient, préconscient et inconscient. À travers le Moi, les humains peuvent avoir une vie et un comportement équilibrés.

Le Ça et le Moi sommes liés aux pulsions. Dans The Ego and The Id (1923), Freud a expliqué qu'il y avait deux classes de pulsion, à savoir le nombre de la mort appelé Thanatos et la vie appelé Eros. Thanatos est la base des attitudes destructrices de l'homme, qui sont des instincts qui mènent au suicide ou tuent d'autres personnes. Eros est la base du maintien du Ça. Eros est associé à des impulsions sexuelles appelées libido et à des impulsions non sexuelles appelées alimentation, liées au désir de manger et de boire (Milner, 1992, p. 105).

\section{Résultat et discussion}

\subsection{Thérèse Raquin : développement de la personnalité}

Thérèse Raquin a deux protagonistes qui font avancer le cours de l'histoire, à savoir Thérèse et Laurent. Outre les deux figures déjà mentionnés, il existe plusieurs autres figures, le mari et le cousin de Thérèse, Camille; Mme Raquin, mère de Camille et tante de Thérèse; Michaud, commissaire de police du vieil ami de madame Raquin; Olivier, fils de Michaud; Suzanne, la femme d'Olivier; et Givet, l'ami et leader des employés de Camille à la compagnie des chemins de fer d'Orléans, où travaille Camille.

Depuis son enfance, Thérèse a été soignée par sa tante et a grandi comme une personne malade. Elle est obligée de se taire et de ne pas bouger beaucoup. Si elle bougeait trop, elle aurait un problème avec sa tante. 
'Sa tante lui avait répété si souvent : "Ne fais pas de bruit, reste tranquille", qu'elle tenait soigneusement cachées, au fond d'elle, toutes les fougues de sa nature. (...) elle fait des mouvements adoucis, des silences, des placidités, des paroles bégayées de vieille femme.'(Thérèse Raquin, Émile Zola, 31)

La citation ci-dessus montre que l'enfance de Thérèse dominée par des règles extérieures à lui-même, que Freud a qualifiées du Surmoi. Les désirs les plus profonds de Thérèse ne peuvent jamais se réaliser. Selon Freud dans The Ego and The Id (1923), pour avoir l'équilibre de la vie, nous devons avoir un équilibre du Moi. Thérèse n'est pas en mesure de trouver un équilibre entre la poussée impulsive et la pression du Surmoi. Cela fait grandir Thérèse en tant que personne déséquilibrée et sans confiance. La personnalité de Thérèse est remplie d'actions répressives contre ses impulsions par crainte de l'environnement social.

Thérèse entre dans le monde des adultes quand elle a déménagé pour la première fois à Paris avec sa famille après avoir épousé Camille. Le mariage qui est aussi une forme de domination de sa tante sur elle.

'Pendant une grande semaine, la boutique et le logement restèrent en désordre. Dès le premier jour, Thérèse s'était assise derrière le comptoir, et elle ne bougeait plus de cette place. (...) Lorsqu'elle proposait une réparation, un embellissement quelconque :

À quoi bon? Répondait tranquillement sa nièce, Nous sommes très bien, nous n'avons pas besoin de luxe.' (Thérèse Raquin, Émile Zola, 42-43)

La citation ci-dessus montre que la personnalité de Thérèse a tenté d'atteindre l'équilibre, en soulignant la rationalité. Pendant ce temps, tous les samedis soir, la famille de Raquin dînait avec les vieilles connaissances de Mme Raquin et l'amie de Camille qui travaillait également à l'usine. C'étaient Michaud, le commissaire de police du vieil ami de Mme Raquin ; Olivier, fils de Michaud ; Suzanne, la femme d'Olivier ; et Givet, l'ami et leader des employés de Camille à la compagnie des chemins de fer d'Orléans, où travaille Camille. Dans ce roman, Thérèse est décrite comme n'ayant jamais aimé l'activité nocturne de jeudi. Elle attendait toujours les clients, il a donc une raison de se rendre au magasin et de servir les clients. Ensuite, elle s'attardera jusqu'à ce que Camille le cherche.

Les inconvénients des routines du jeudi soir peuvent être classés dans la catégorie anxiété. Le Ça de Thérèse veut du réconfort en ne voyant pas d'invités dégoûtants, comme décrit dans le roman. Cependant, le Surmoi sait qu'il ne s'agit pas d'une attitude polie d'un hôte vis-à-vis de ses invités, ni d'une attitude positive pour son mari et sa tante. Alors même si elle voulait rester plus longtemps dans le magasin, elle obéissait quand Camille l'appelait dans les escaliers. Il y a eu un changement dans Thérèse, qui a commencé à oser écouter de son Ça et n'était plus dominé par Surmoi. On peut interpréter que c'est la période où Thérèse a un équilibre du Moi en lui. L'activité du jeudi soir est aussi une description de l'aliénation de Thérèse du monde dans lequel elle vit. Les quatre personnes qui sont venues au programme du jeudi soir étaient des connaissances de Mme Raquin et des connaissances de Camille. Thérèse n'avait pas d'amis pour la routine du jeudi soir. C'était probablement le résultat de son attitude passive qui avait été plantée depuis son enfance, Thérèse a grandi sans avoir d'amie.

Laurent était un vieil ami de Camille, qui l'avait souvent accompagné à l'école. Ils se retrouvèrent à l'usine où travaillait Camille, puis Camille l'invita à rencontrer madame Raquin et Thérèse. Zola a décrit la figure de Laurent comme un homme musclé et fort, tout le contraire de la fragile Camille. La figure de Laurent est ensuite remarquée par Thérèse, une femme qui ne s'associe pas à d'autres personnes, en particulier à un homme. Cette rencontre a provoqué un choc différent chez Thérèse, elle a senti la passion qu'elle n'avait jamais eue avec Camille.

Laurent lui-même est décrit comme une personne égoïste et veut toujours avoir un plaisir instantané. Il a trompé son père en abandonnant ses études et a ensuite utilisé cet argent pour louer des mannequins nus en tant qu'objets de peinture ainsi que des objets pour satisfaire ses désirs sexuels. Le comportement de Laurent est influencé par son désir du plaisir. Des gens comme Laurent sont l'image parfaite d'une personnalité dominée par Ça.

Quand il a commencé à entrer dans la vie de la famille de Raquin, ses désirs de plaisir ou ce que Freud appelait Eros, ont commencé à réaffecter sa personnalité. . Au début, il était si amical et poli qu'il était bien accueilli par la famille Raquin. Tout a changé quand Laurent a commencé à voir Thérèse comme une femme séduisante. Son intérêt pour Thérèse s'est transformé en passion pour la possession.

Thérèse, elle aussi qui, au début, elle a montré l'hospitalité et la courtoisie à Laurent. Elle n'a pas quitté la salle à manger lors de l'événement de jeudi soir pour faire attention à Laurent mais elle n'a pas osé s'adresser à lui. Tout s'est terminé quand Laurent a finalement osé embrasser Thérèse. 
Le mur des normes sociales de Thérèse s'est effondré et son subconscient a éclaté bruyamment.

'Puis, d'un mouvement violent, Laurent se baissa et prit la jeune femme contre sa poitrine. Il lui renversa la tête, lui écrasant les lèvres sous les siennes. Elle eut un mouvement de révolte, sauvage, emportée, et tout d'un coup, elle s'abandonna, glissant par terre, sur le carreau. Ils 'n'échangèrent pas une seule parole. L'acte fut silencieux et brutal.'(Thérèse Raquin, Émile Zola, 72)

Cette scène est le moment où Laurent est vraiment entré dans la vie de Thérèse et vice versa. Les deux sont dominés par Ça sous forme de pulsion Eros. Le désir sexuel qui peut être accompli l'un avec l'autre a vaincu leur Surmoi. Laurent se fichait que Thérèse fût la femme de son ami ; elle ne se demande plus si ses gestes nuiront aux sentiments de son mari et de sa tante. Zola a déclaré en disant le mot «brutal » comme s'il décrivait que l'acte n'était pas comme un acte humain civilisé. Ceci est conforme à ce que Freud a expliqué : le C'est une attitude primitive de l'homme qui est apparue pour la première fois chez les individus.

'Il avait des heures d'effrois, des moments de prudence et, en somme, cette liaison le secouait désagréablement; mais ses peurs, ses malaises tombaient devant ses désirs. Les rendez-vous se suivirent, se multiplièrent' (Thérèse Raquin, Émile Zola, 77)

L'équilibre du Moi de Laurent commença aussi à s'effacer sous l'impulsion de son Ça pour satisfaire sa convoitise avec Thérèse. Ils ont même osé le faire dans la chambre de Camille et Thérèse. Une fois, Mme Raquin est entrée dans la pièce quand Laurent était là, mais Thérèse a réussi à la piéger en faisant semblant d'être malade et en cachant Laurent sous sa chemise de nuit. Auparavant, il était dit que Thérèse était une personne soumise, surtout à sa tante. Mais quand Laurent est entré dans son monde et qu'il a commencé à être dominé par Id, alors elle a osé mentir à sa tante.

Les pulsions sexuelles qui avaient maitrisé Laurent et Thérèse les ont rendues agitées car elles ne pouvaient pas la canaliser librement. Deux personnes dominées par cette convoitise libidinale ont vu la figure de Camille comme une barrière à supprime. Sans hésiter, ils ont alors conçu un plan pour tuer Camille. Thérèse, au départ effrayée et hésitante, a reçu un soutien équilibré de Laurent qui avait tendance à être plus rationnel. Lorsque Thérèse plus entraînée désir de Laurent, puis Laurent ont également des motifs économiques pour le contrôle de la fortune de la famille Raquin. Et un jour, Thérèse et Lauren ont réussi à éliminer Camille en concevant la mort de Camille comme un accident.

La mort de Camille n'a pas donné la tranquillité à Thérèse et Laurent. Ils souffrent d'une énorme culpabilité. Ils sentaient que l'esprit de Camille les suivait toujours et se vengerait. L'émergence de ces sentiments de culpabilité montre que le facteur du surmoi a toujours un impact sur la personnalité de Thérèse et de Laurent. Leurs efforts pour répondre à l'impulsion du Ça les ramène à la pression du Surmoi.

\section{Conclusion}

Le roman de Thérèse Raquin a de fortes caractéristiques en tant que roman de naturalisme. Le naturalisme est la suite logique du réalisme qui essayait de décrire la réalité de la manière la plus précise possible, y compris dans ses aspects immoraux ou vulgaires. Le naturalisme ajoute un contexte physiologique et montre que le milieu où vit le protagoniste est l'une des raisons de son comportement.

Thérèse en tant que personnage principal est décrite comme ayant une personnalité influencée par son environnement social. En tant que personnalité, Thérèse est incapable d'équilibrer ses impulsions avec la pression des normes sociales ou du Surmoi. Les désirs personnels qu'elle a toujours réprimés, ont finalement explosé suite à son amour pour Laurent, même s'elle avait épousé Camille. L'impulsion du Ça a ensuite maîtrisé chaque action de Thérèse jusqu'à ce qu'il atteigne son apogée avec le meurtre de Camille. Mais la domination du Ça ne dure qu'un moment. La culpabilité a violé la plus grande norme sociale en commettant le meurtre de Camille, laissant Thérèse incapable de sortir de la pression du Surmoi. Cela montre que les facteurs sociaux sont la partie la plus décisive du roman de Thérèse Raquin d'Emile Zola.

\section{Références}

Freud, S. (1960). The Ego and The Id. New York: W.W Norton \& Company. 
Klarer, M. (2004). An Introduction to Literary Studies (2nd ed.). London: Routledge.

Milner, M. (1992). Freud dan Interpretasi Sastra. (S. W. Apsanti \& P. Laksmi, Trans.). Jakarta: Intermassa. Shivanandan, M. (2007). Emile Zola: Improbable Defender of Life. In Life and Learning XVII: The

Proceeding of Seventeenth University of Life Conference (pp. 509-527). New York: University Faculty for Life. Retrieved from www.uffl.org/vol17/SHIVANANDAN07 